\title{
Interactive comment on "Evidence of coastal trapped wave scattering using high-frequency radar data in the Mid-Atlantic Bight" by Kelsey Brunner and Kamazima M. M. Lwiza
}

\section{Kamazima Lwiza}

kamazima.Iwiza@stonybrook.edu

Received and published: 21 August 2020

1. When the reviewer states that, 'CTWs and their dynamics being proposed as the sole and only possible explanation for the observed surface currents features, the -very large- discrepancies between observations and this theoretical framework being systematically ascribed to "scattering" processes of the CTWs.' They are overlooking the fact that in the methods section we describe how we bandpassed the current signal between 3 and 12 days. Therefore, we are excluding all the small scale (temporal) processes including signals caused by some of the short synoptic scale weather forcing. The next statement regarding the propagation of order- 0 , with the scattered signal 
translating into higher orders is in fact demonstrated by showing that energy scattered from mode 1 into modes 2 and 3. Also, not finding other papers describing this phenomenon should not be the basis to refute its (scattering) existence. It must be noted observation of coastal trapped waves of the type we describe have only been made possible by the advent of HF radar. Therefore, it should not be surprising that this is the first paper that provides such evidence (see a comment by John Huthnance, one of the people who pioneered observations and theory of CTW).

2. The second issue is on the reviewer being skeptical of the use of the C-EOF method in extracting propagating signals. We kindly request the author to read the literature using this method and some of the references we provide in the manuscript. It is also used extensively in Atmospheric Physics. In addition, the reviewer can easily use matlab or python and create a propagating field with some noise and try out the CEOF and compare it to what he proposes [u du/dt] ( I have ignored the v component for simplicity). I tried the comparison, and C-EOF reproduces the phase very well, except where the signal is almost equal to the error, whereas the proposed method gets the general phase pattern, but broadens the peaks. In any case, the state of the reviewer being unfamiliar with a method should not be a criterion to disqualify the method or the manuscript.

Interactive comment on Ocean Sci. Discuss., https://doi.org/10.5194/os-2020-46, 2020.
OSD

Interactive comment
Printer-friendly version

Discussion paper 


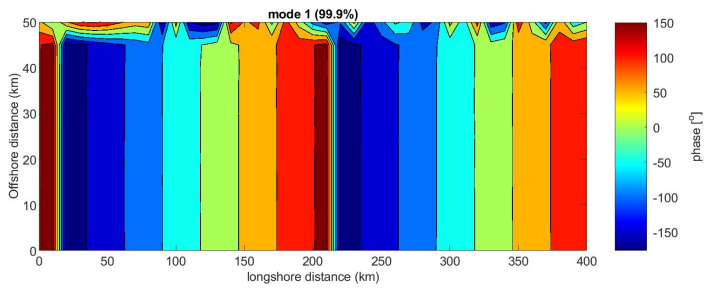

\section{Interactive comment}
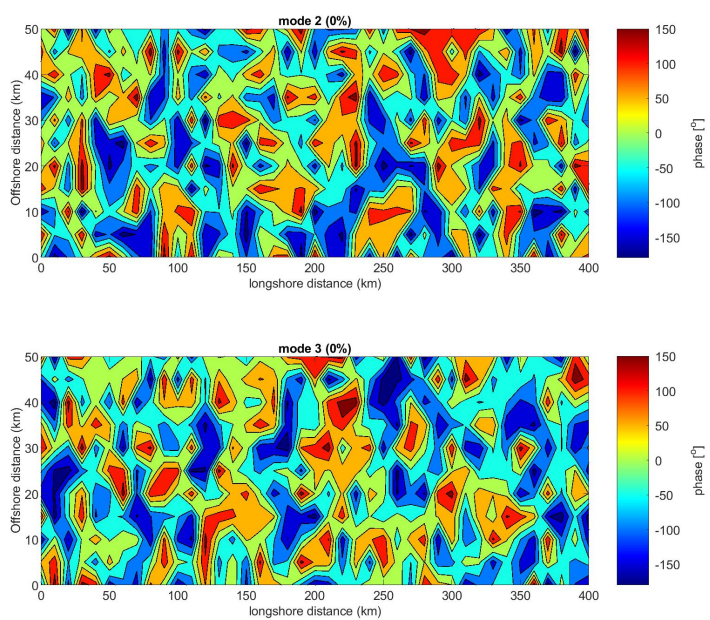

Fig. 1. Complex EOF of simple propagating wave signal from left to right with a linearly decreasing amplitude offshore 
OSD

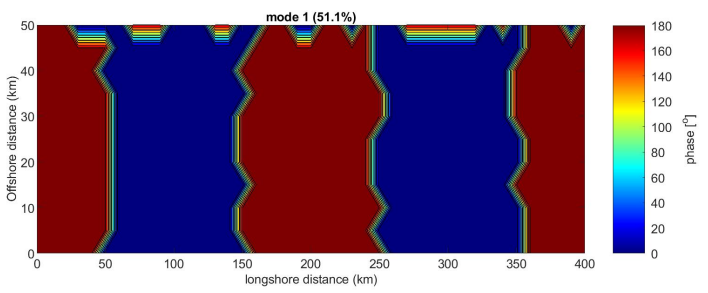

Interactive comment
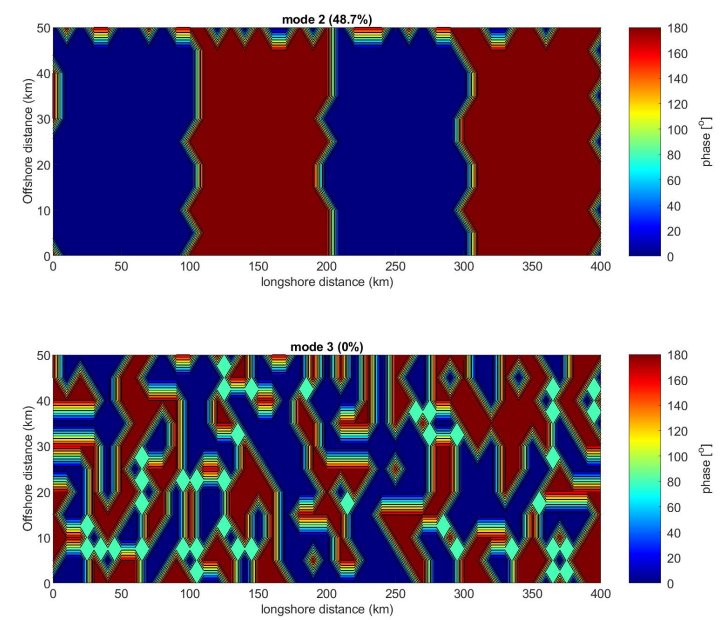

Fig. 2. Real EOF with [u du/dt] as suggested by Reviewer\#1 everything else similar to Fig. 1 